\title{
System Architecture Modeling of an UWB Receiver for Wireless Sensor Network
}

\author{
Aubin Lecointre, Daniela Dragomirescu and Robert Plana \\ LAAS-CNRS \\ University of Toulouse \\ 7, Av du Colonel Roche \\ 31077 Toulouse cedex 4, France \\ \{alecoint, daniela, plana\}@laas.fr
}

\begin{abstract}
This paper presents a method for system architecture modeling of an IR-UWB (Impulse Radio Ultra WideBand) receiver for sensors networks applications. We expose the way for designing an FPGA (Field Programmable Gate Array) receiver starting from a previous study based on system modeling on Matlab. The proposed receiver architecture is first designed and validated on Matlab, before being implemented, thanks to VHDL language, on a FPGA. Our study shows the interest and the advantages of co-design Matlab-VHDL. We will propose here different IR-UWB receiver architecture depending on the modulation used. We will also introduce in this paper a data-rate and TH-code reconfigurable receiver. Using co-simulation Matlab-VHDL, we have compared three kind of IR-UWB receiver: TH-PPM, TH-OOK, TH-BPAM, with respect to BER/SNR criteria and in the specific context of wireless sensors networks, at high level (Matlab) and hardware level (FPGA-Xilinx).
\end{abstract}

\section{Introduction}

We lead our study in the context of wireless sensors networks (WSN). We define WSN as systems having a very large number of nodes on a small area. WSN is a WPAN-like concept (Wireless Personal Area Networks). There are a lot of kinds of applications for this variety of networks; such as: monitoring, military applications, house automation, civil safety applications, etc ... By considering these applications, we could deduce easily that there are some intrinsic constraints for WSN, which are: low cost, low power, simplicity and tiny nodes. Indeed, without theses characteristics none networks could be a viable WSN. Thus all along this paper we keep in mind this context in order to design and compare in an appropriate way the UWB receivers.

The Federal Communications Commission (FCC) defines a radio system to be an UWB system if the $-10 \mathrm{~dB}$ bandwidth of the signal is at least $500 \mathrm{MHz}$ or the fractional bandwidth is greater than $20 \%$ [1].

IR-UWB is a very promising technology for the WSN applications. Let us quote these advantages: $7,5 \mathrm{GHz}$ of free spectrum which could permit to reach high data rate, extremely low transmission energy, extremely difficult to intercept, multi-path immunity, low cost (mostly digital architecture), "Moore's Law Radio" 
(performances, size, data rate, cost follow Moore's Law), simple CMOS transmitter at very low power [2]. Among the various families within UWB, we focus on family IRUWB, Impulse Radio UWB which is appropriate for our context of application: wireless sensor network. The principal modulation techniques we will use are: Time Hopping - Pulse Position Modulation (TH-PPM), Time Hopping - On Off Keying (TH-OOK), and Time Hopping - Binary Pulse Amplitude Modulation (TH-BPAM). We will study these three major IR-UWB techniques: TH-PPM, TH-OOK, and THBPAM [1]. For each of them we will propose one or more schemes of receivers. We will present theirs design and co-simulation using Matlab and ModelSim. Our goal is to develop and validate, at first, the receiver architecture at a high level using MATLAB. Then, we would reach the low-level of hardware simulation and implementation, i.e. the FPGA development.

Finally, we would compare theses different systems, including data rate and TH-code reconfigurable receiver, according to the BER (Bit Error Rate) versus SNR (Signal Noise Ratio) criteria and with respect to the WSN constraints.

This paper is organized as follows: Section II presents the principle of TH-OOK, THPPM, and TH-BPAM, as well as theirs high level modeling on Matlab. Section III describes the design and the implementation of the UWB receiver on the FPGA. We will compare TH-PPM, TH-OOK, TH-BPAM architectures and performances in section IV, before conclusion in the section V.

\section{High level modeling of UWB Transceivers}

\subsection{Principle of Pulse Modulation for Time Hopping IR-UWB}

TH looks like a dynamic TDMA [3]. TH consists of the sharing of the medium in the frame. Each frame is divided in time slots. TH allows making multi user communications. The repartition of information depends of the time hopping code which is associated with each user. Once slots are defined, we could apply the pulse modulation either PPM or OOK or BPAM.

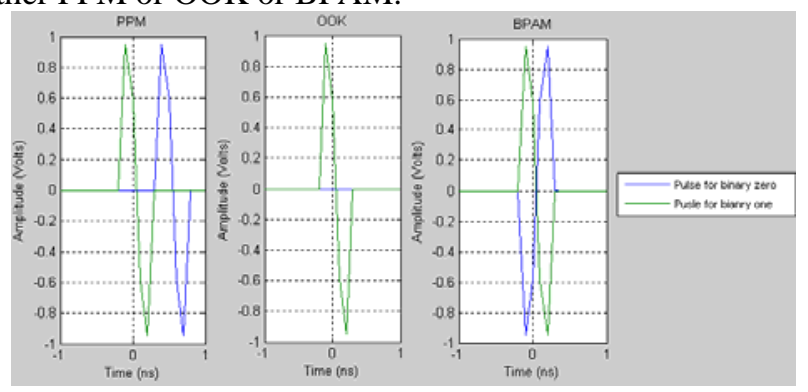

Fig. 1. IR-UWB Modulation: PPM, OOK, BPAM

For the PPM, bits are differed by a time shift in each time slot selected by the TH code. Concerning the OOK, we send a pulse in the slot for transmitting a binary one. 
The binary zero will correspond to an absence of impulsion. For the BPAM, the binary one and the binary zero differ by the phase of the pulse. Thus, the binary zero is represented by the binary one pulse multiplied by minus one $\left(-1 \Leftrightarrow 180^{\circ}\right)$ (Fig. 1).

\subsection{High Level Modeling on Matlab for TH IR-UWB Emitters}

The implementation of these three TH systems on Matlab is based on a high-level modeling. We have developed a complete link, from emitter to receiver including channel model. Our Matlab model is parametric, so we can select the kind of IRUWB we want to study among TH-PPM, TH-OOK, and TH-BPAM. This choice will impact both end (emitter and receiver) of the IR-UWB link.

Thanks to the time domain approach of IR-UWB, emitters are very simple. Indeed, it is enough to implement an UWB pulse generator which is commanded by a binary signal where binary one and binary zero have a specific meaning according to the IRUWB modulation considered. They impose, for example, the amplitude, or the position of modulated pulse.

At the output of these receivers, the IR-UWB signal is sent over a channel. This latter could be an AWGN (Additive White Gaussian Noise) channel or the IEEE 802.15.4a UWB channel [4], [5]. Receivers follow the channel. There are different receivers in function of the IR-UWB technique employed.

\subsection{High Level Modeling on Matlab for TH IR-UWB Receivers}

For TH-OOK, we propose a non-coherent receiver described in figure 4 [6]. This non coherent architecture is composed of four blocks: a filter on the considered band, a square bloc, an integrator bloc, and a decision bloc. Its principle is energy detection. The received signal is squared before being integrate. Consequently there is no need of synchronization mechanism; this confers the simplicity advantage at this architecture [6]. That's why this receiver is less expensive, simpler, less greedy in power consumption, and it has smaller overall dimensions than the TH-PPM receiver.

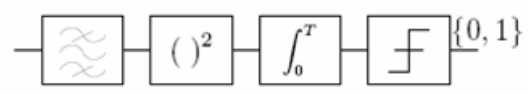

Fig. 4. Non coherent OOK receiver

As described on figure 5, the TH-PPM coherent receiver is based on the correlation, with a template waveform, principle. The receiver generates a pulse whose form must be as far as possible like the received pulse. This should allow reaching better performances. The nearer the template waveform looks like the received pulse, the better the performances are. Once the template is generated, the correlation between the template and the received pulse is carried out. The concept is to compare the received pulse with the expected pulse corresponding to a "one" or a "zero". The higher the resemblance with a "one" template is, the probability that the received pulse is a one logic, is more important. At the output of the two correlation blocs (one 


\section{Aubin Lecointre, Daniela Dragomirescu and Robert Plana}

and zero logic), it is enough to place a comparator with two inputs to distinguish, according to the amplitude, one logic from zero logic.

A synchronization bloc is also necessary, in order to provide a synchronous correlation between the received pulse and the template waveform. This function is carried out by a matched filter defined on a known (emitter and receiver side) sequence of pulse [7]. This sequence has a good autocorrelation property. This filter generates a peak in the presence of a synchronization trailer at its input. Thus, we detect the peak, thanks to a comparator, and we have the time arrival of the pulse. As the result we are able to synchronize the receiver. This synchronization is difficult, because of the pulse duration ( $<1 \mathrm{~ns})$ and should be the most precise possible, otherwise the correlation output will be always at zero, and so the received bit will be also always at zero.

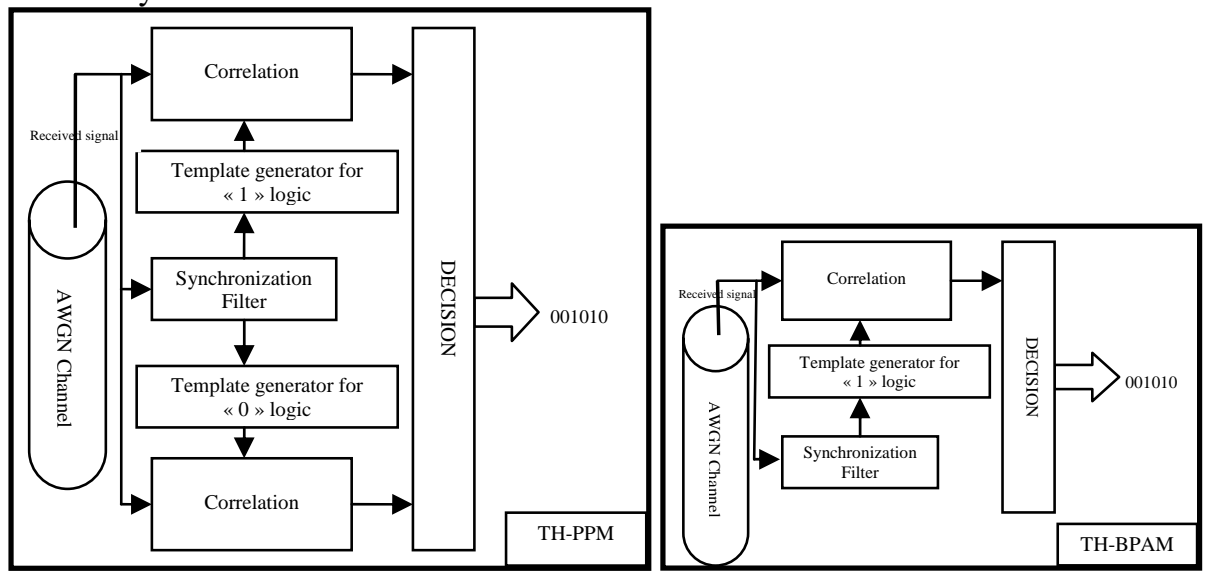

Fig. 5. TH-PPM coherent receiver and TH-BPAM coherent receiver

Figure 5 and 6 illustrate the TH-BPAM receiver. Its concept is very similar to the THPPM receiver. Indeed, the correlation principle is employed in order to determine the state of received data. As correlation is used, synchronization mechanism is also required.

For TH-BPAM receiver, we propose to use only one correlation with template, instead of two correlation proposed in TH-PPM receiver [1]. Since the BPAM pulse representing the binary one is the inverse of the pulse for the binary zero, if we use only one correlation block, we will have at its output, either a positive squared impulse or a negative squared impulse. This simplifies the decision, because at the output of the correlation block binary one and binary zero could be distinguished by the polarity of the signal.

Compare to TH-PPM and TH-OOK architecture, this one seems to be an intermediate solution. Indeed, thanks to the use of only one correlation block, a simpler decision, this receiver is simpler, cheaper, smaller and have a lower energy consummation than TH-PPM receiver. Nevertheless, the TH-OOK receiver remains the reference concerning the principal WSN constraints (cf. table 1). We will decide, in the next part, between these receivers, according the BER versus SNR criteria. 


\subsection{Comparative Analysis}

Table 1 summarizes the behavior of each IR-UWB proposition in the WSN context.

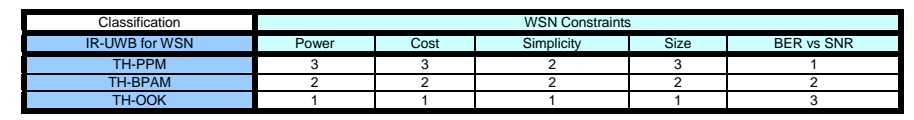

Table 1. Comparative analysis of IR-UWB architectures.

We could note that size, cost, and power constraint seems to be linked. This is logic, since the increase in components number will increase the cost and the energy needs. So, we have three possibilities: TH-PPM, TH-BPAM, TH-OOK in order to answer WSN context. The most adequate, without taking into account the BER performances, is TH-OOK, followed by TH-BPAM, and then TH-PPM.

The final decision should be taken considering, the BER versus SNR criteria. There is a compromise between respect of the WSN constraints and BER performances.

In order to use the BER versus SNR criteria, we have used an AWGN channel model in our Matlab modeling. The figure 6 shows this BER comparison [8].

This curve illustrates the classification of the last column of the table 1 . We could observe that the TH-PPM propose better performances, a gain of about $6 \mathrm{~dB}$, than the TH-BPAM and $8 \mathrm{~dB}$ than TH-OOK. This allows us to notice that there is a trade-off between BER performances and WSN constraints criteria.

Figure 6 proves also that IR-UWB techniques offer better performances than continuous wave (CW) modulation (FSK, PSK QAM). Indeed, figure 6 permits us to quantify the gain when we use IR-UWB systems instead of CW techniques; its value is about $40 \mathrm{~dB}$.

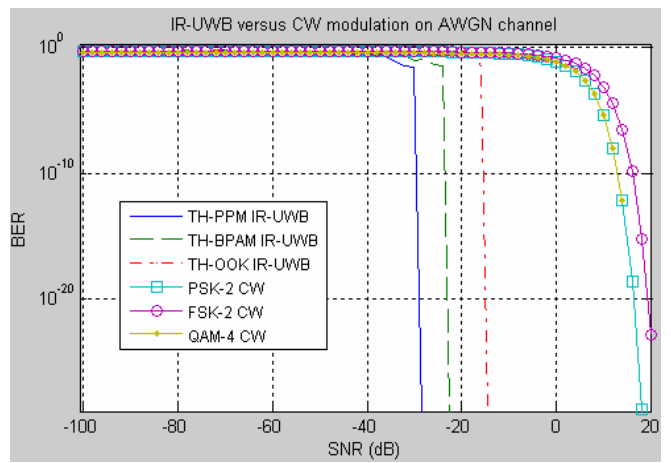

Fig. 6. IR-UWB versus continuous wave, according to BER/SNR criteria, on AWGN channel.

In order to conclude, we can say that our high level modeling with Matlab shows:

- on one hand that IR-UWB is very interesting in the WSN context, because of its adaptability to the four WSN constraints and its better BER performance than classical CW techniques.

- on the other, our Matlab modeling validates the different architectures in terms of viability for WSN.

As a result, after this essential phase, we could begin the FPGA implementation and simulation, i.e. the hardware-level study. 


\section{FPGA Design of an UWB receiver}

\subsection{The Low-level Modeling Context}

For implementing an IR-UWB receiver we have decided to use Xilinx Spartan III FPGA, because it is a cheaper and a optimized signal processing solution. We have chosen Xilinx software solution for designing and simulating our receiver. Nevertheless, since we won't set up the emitter on the FPGA, we will use Matlab in order to emulate the comportment of the channel and of the emitter. Moreover we use also the platform to simulate the MAC (Medium Access Control) layer, that is to say the layer which is responsible for piloting the receiver at the PHY (PHYsical) level. Before exposing our low level developing platform, note that we don't consider the RF stage at hardware level. The RF stage, as well as the channel, will be simulated by MATLAB. Thus, as we can see on the figure 7, each element is designed in baseband, behind the ADC for the receiver.

Figure 7 shows the collaboration work between the computer and the FPGA. The computer allow us to develop and program the FPGA, then it is used for emitter plus channel emulation, and finally, it permit to estimate the BER, thanks to received data which come from the FPGA.

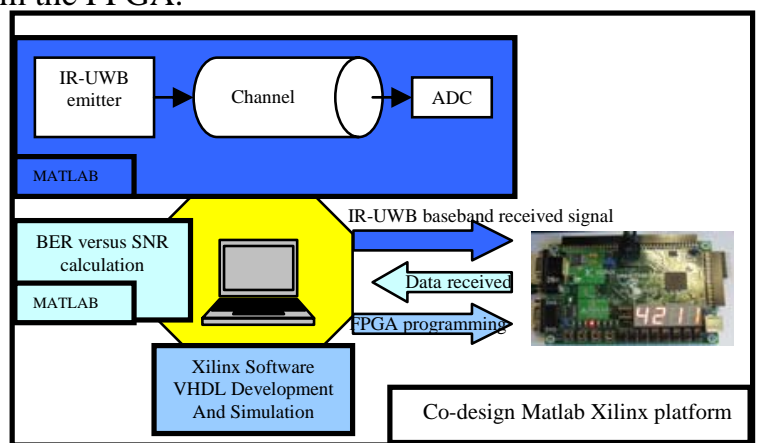

Fig 7. Co-simulation and co-performances analysis Matlab Xilinx Platform.

\subsection{FPGA Implementation with VHDL.}

VHDL (Very-high-speed integrated circuit Hardware Description Language) is the design programming language we use for digital circuits. We designed our three receivers (TH-OOK, TH-PPM, TH-BPAM) in VHDL according to a modular concept. We designed each of the elementary blocs in charge of elementary receiver function such as multiplication, TH-discrimination, TH code management, decision... Using this kind of modular design, we can propose easily different versions of each kind of receiver. For each IR-UWB receiver, i.e. TH-PPM, TH-OOK, TH-BPAM, we have created different solutions in order to answer to the four WSN constraints at different levels. For example, one version could be greedier in energy consumption 
but in return it should have, for example, better BER performance or an additional function. As the opposite, we could imagine some light versions, which could have as goals to offer correct BER performance but especially optimize the four other constraints: power, size, cost, simplicity. Thus we have distinct receiver versions more or less complex, more or less performing, more or less greedy in energy, etc ... Our panel of receivers is configurable according to four main parameters:

- number of bits used for representing IR-UWB signal,

- number of TH logic channel implemented,

- the presence or not of the localization module,

- the fact that receiver properties are static, or reconfigurable.

Note that we will discuss in the next section (§ 3.3) on the concept of static and reconfigurable properties.

\subsubsection{TH-OOK receivers}

We have seen in the section II that TH-OOK is the low power, low cost, smallest, and simplest solution among TH-PPM and TH-BPAM. Thus, it seems to be logic to propose an optimized TH-OOK receiver. That's why we have implement on our FPGA a simple solution, whose characteristic are: mono channel reception, absence of localization mechanism, static properties, and 64 bits processing. Let us call this version TH-OOK-v1.

TH-OOK-v2 consists of practically TH-OOK-v1 except that this second version work on 32 bits. Figure 8 exposes the architecture of TH-OOK-v1 and TH-OOK-v2. We can consider that TH-OOK-v2 is an optimized receiver for ultra low cost, ultra low power, ultra small and ultra simple WSN applications.

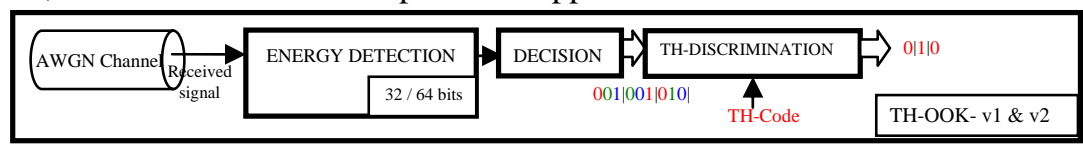

Fig. 8. FPGA implementation of TH-OOK receiver.

Let us benefit from this illustration (figure 8) to explain the concept of $\mathrm{TH}$ discrimination. Previously, we have introduced the TH concept, by recalling that the channel was divided in frame and time slot. One TH-code is allotted per user or per communication. TH-code defines which time slot will be used by the user or the associated communication. As a result, TH-discrimination consists in extracting the information corresponding to the considerate TH-code among the multi TH-code signal. So, we need a TH-code for the discrimination.

Thanks to this TH-discrimination notion, we could apprehend the mono channel or double channel receiver. Some WSN need an information data channel and a control data channel, or also, multi-user channel. Thus WSN receivers must be able to deal with several "channels". This implies they must be able to extract several THchannels from a multi-channel flow; so TH discrimination bloc should have as many TH-code entries as there are channels to receive.

\subsubsection{TH-BPAM receivers}

TH-BPAM receiver versions, TH-BPAM-v1 and TH-BPAM-v2, are represented on figure 9. These two versions allow us to analyze the importance of the blocs' position. 

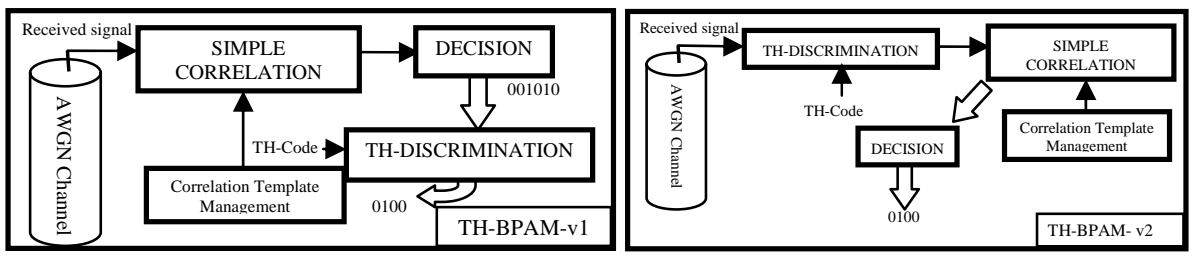

Fig. 9. FPGA implementation of TH-BPAM-v1 and TH-BPAM-v2 receiver.

Indeed, we have changed the position of the TH-discrimination bloc in the second version in comparison with the first one. Note that we could easily invert the blocs' positions, thanks to the modularity conception principle. These TH-BPAM receivers, based on the simple correlation concept, are mono channel, static, and work with 32 bits. They don't implement distance estimation and localization.

\subsubsection{TH-PPM receivers}

We have implemented the distance estimation in one of the TH-PPM FPGA receiver versions. Theirs architectures follow the figure 5, i.e. the Matlab TH-PPM double correlation coherent receiver. We will use four versions:

- TH-PPM-v1: mono channel, static properties, 32 bits, without distance estimation.

- TH-PPM-v2: mono channel, static properties, 32 bits, with distance estimation.

- TH-PPM-v3: mono channel, reconfigurable, 64 bits, without distance estimation.

- TH-PPM-v4: double channel, reconfigurable, 64 bits, without distance estimation. We have chosen these four versions in order to be able to examine the impact of: size of sample, reconfigurability, distance estimation, and multi channel aspect, on the IRUWB receiver according to the WSN constraints.

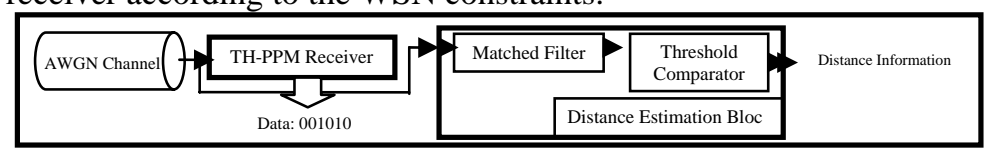

Fig. 10. FPGA implementation of TH-PPM-v2 receiver with distance estimation mechanism

Fig. 10 presents the TH-PPM-v2 receiver architecture with localization mechanism. Some WSN applications want to be able to geo-localize each node reciprocally to optimize the network routing. To estimate the position of the emitter, the receiver must be able to evaluate the time of arrival of the received pulse. Using this information, the receiver could determine the distance separating the emitter from the receiver, since the celerity of the pulse over the air is known. For obtaining this arrival time, we use at the entrance of the receiver, a matched filter (figure 10). Its output is maximal when we are at the time arrival of the pulse [9]. Thus we have just to add a threshold comparator to detect this maximum, for determining the arrival time and consequently the distance.

As the opposite of TH-OOK-v2 receiver, we can say that TH-PPM-v4 is a suitable receiver for the most of WSN applications while offering good BER/SNR performances. Nevertheless it couldn't be considered as a WSN constraints full optimized receiver. 
Further, we will establish low level BER/SNR performances in order to confirm the high level modeling results obtained with Matlab.

By proposing different versions of our IR-UWB receivers, we would like to expose our two reflexion way as response for WSN problematic:

- either we create an optimized radio interface for each main category of WSN;

- or we will direct ourselves toward a kind of absolute solution, whose the goal would be to adapt to any WSN applications needs.

This second way is the most innovative way, and it proposes the implementation of reconfigurablity concept inspired from software-defined radio [10].

\subsection{Data Rate and Time Hopping Code Reconfigurable Receiver.}

In this part, we will present the reconfigurable aspect of our system. We consider two type of properties receiver reconfigurability

- Static properties: absence of reconfigurability. Receiver characteristics values, such as the TH-frame duration, number of time slots per TH-frame, TH-code, TH-time slot duration, etc ... are registered in hard in the VHDL code. Thus for adapting our reception system, we modify the code, and re-download it in the FPGA.

- Reconfigurable properties: it is the most accomplished of our receiver according to the radio reconfigurability concept. Receiver characteristics values are modifiable without re-program the FPGA. We implement that in TH-PPM-v3 and v4 receivers by means of MAC-layer entries. This kind of reconfigurable receiver has many applications in self-organizing WSN where the data rate can be very variable.

Modifying the Time Hopping properties, (number of slot per frame, frame duration, time slot duration), leads to data rate change. Since the data rate, on the whole Time Hopping link (considering all the possible TH-code), depend on the frame duration (Tf), the time slot duration (Tc), and the number of time slot per frame (Nc).

$$
\mathrm{D}_{\text {total }}(\text { bits } / \mathrm{s})=\mathrm{N}_{\mathrm{c}} / \mathrm{T}_{\mathrm{f}}=\mathrm{Nc} /\left(\mathrm{N}_{\mathrm{c}} \times \mathrm{T}_{\mathrm{c}}\right)=1 / \mathrm{T}_{\mathrm{c}} .
$$

The TH-PPM-v4, mentioned previously, is a data rate reconfigurable receiver.

During a transmission between two nodes of a WSN, one of them decides to change its data rate; the second is able to modify also its data rate, in order to continue the communication. This possibility of data rate modification is an advantage in the concurrent context of channel access in WSN. We will note that it is upper layer protocol, such as MAC layer and applications layers, which are responsible for selecting the best moment to commute the data rate.

Furthermore, in our reconfigurable receiver, we have also implement TH-code reconfiguration. It consists of being able to change the TH-code reception during the communication and consequently the received channel.

In order to set up this reconfiguration concept, we have implanted the reconfigurable parameters as MAC layer entries (fig. 11). The MAC layer emulated by the computer thanks to Matlab, or Xilinx software, is in charge of:

- sending the configurable parameters to the FPGA

- start the reconfiguration by sending a signal, called "reconfiguration signal". 
As MAC layer is an intelligent organ we could make the supposition that it sends the "reconfigurable signal" only after correctly place reconfigurable parameters at entries.

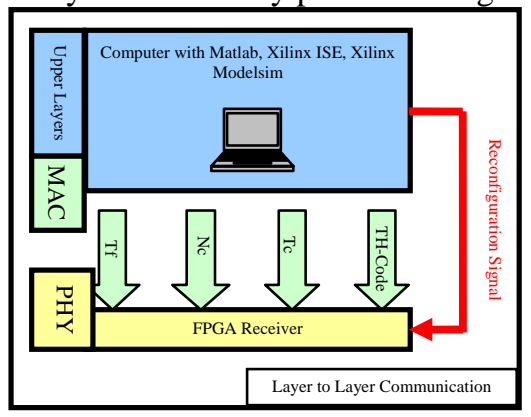

Fig. 11. PHY and MAC Layer interface.

Our radio reconfigurability design has some limitations. Indeed, as we use VHDL entity entries for reconfigurability, we undergo theirs limitations. In our example, we choose 8 bits to implement each reconfigurable parameter. It implies that we couldn't reach any kind of data rate in the reconfiguration (without re-programmed the FPGA). Nevertheless, this is true only if we don't take into account the RF limitations (due to RF circuits). In fact, it is this one, which will limit the data rate. Consequently, the VHDL entries limitations sizes (when FPGA is programmed) is not a limit but rather a dimensioning preoccupation. Since, once the FPGA is programmed we would be limited by the defined maximum value of your distinct entries. This dimensioning is important because the number of bits allocated impact the size and consumption of the receiver, which are two important constraints in our WSN context.

In the following part, we will make a comparative analysis of our receivers; we will demonstrate the relation between the number of bits used and the size of the receiver.

\subsection{FPGA Receiver Performance}

In this part, we will compare eight versions of IR-UWB receiver according to the BER versus SNR criteria, and the four WSN constraints: energy consumption, size, cost, and simplicity. Besides, this comparative analysis should highlight the impact of change in VHDL implementation: number of bits for processing, limit size (in bits) for the VHDL variable, presence/absence of distance estimation or radio reconfigurability, and the number of received channel.

\begin{tabular}{|c|c|c|c|c|c|c|c|c|c|}
\hline & \multicolumn{5}{|c|}{ Receiver Propertie } & \multicolumn{3}{|c|}{ WSN Constraints } & $\operatorname{man}$ \\
\hline VERSION & Receiver Principle & Sample Size & Channel Number & Distance & Radio Reconf & VERSION & Size (in gate) & Max. Frequency & BER/SNR \\
\hline TH-OOK-v1 & \begin{tabular}{|l|} 
Energy Detection \\
\end{tabular} & 64 bits & Mono Channel & NO & Static & TH-OOK-V1 & $86234+864$ & $89,506 \mathrm{MHz}$ & 8th \\
\hline TH-OOK-V2 & \begin{tabular}{|l|} 
Energy Detection \\
\end{tabular} & 32 bits & Mono Channel & NO & Static & \begin{tabular}{|l|} 
TH-OOK-V2 \\
\end{tabular} & $68666+864$ & $1111,693 \mathrm{MHz}$ & 7 th \\
\hline TH-BPAM-V1 & Simple Correlation & 32 bits & Mono Channel & No & tic & TH-BPAM-V1 & $68663+864$ & $111,693 \mathrm{MHz}$ & 5 th \\
\hline & Simple $\mathrm{Co}_{0}$ & $32 \mathrm{bit}$ & Inunue & 10 & & \begin{tabular}{|l} 
TH-BPAM-V2 \\
\end{tabular} & $68716+864$ & $111,693 \mathrm{MHz}$ & 5 th \\
\hline TH-PPM-V1 & Double Correlation & 32 bits & Mono Channel & NO & Static & \begin{tabular}{|l|} 
TH-PPM-V1 \\
\end{tabular} & $69674+864$ & $110,9 \mathrm{MHz}$ & $1 \mathrm{st}$ \\
\hline TH-P & Double 0 & & el & YES & Static & PM-V2 & 1015 & $\mathrm{MHz}$ & 1st \\
\hline TH.PPM-v3 & Double Correlation & 64 bits & Mono Channel & NO & econfigurable & 1-13 & $107753+864$ & $72,844 \mathrm{MHz}$ & 3rd \\
\hline TH-PPM-V4 & Double Correlation & 64 bits & Double Channel & NO & Reconfigurable & TH-PPM-V4 & $125441+1056$ & $72,844 \mathrm{MHz}$ & 3rd \\
\hline
\end{tabular}

Table 2. Receivers' Characteristics and receivers' comparison.

Table 2 summarizes the receivers' properties and exposes the architecture receiver comparison according to the WSN constraints and the BER/SNR performances. 
In table 2 we expose size and maximum frequency criteria; thanks to them we are able to obtain the four WSN constraints (cost, simplicity, size, energy). Indeed, these four WSN constraints are linked to the size and the maximum frequency of the FPGA circuit. Small circuits mean low cost and low power consumption circuits. The low power property depends also by the maximal frequency. Thus with size and frequency criteria and BER criteria, we could make an interesting classification of receivers. The "size" column gives us a classification between diverse systems. We will note that TH-OOK-v2, TH-BPAM-v1, TH-BPAM-v2, and TH-PPM-v1 are the smallest receivers. Their common point is the simplicity of theirs architecture and the fact that they use 32 bits for the samples coding. Whereas most of 64 bits architectures THPPM-v3 and TH-PPM-v4 are the most cumbersome receiver, in addition with THPPM-v2, which implements distance estimation mechanism. In order to conclude, size is function of the complexity (presence of distance estimation, number of channel, double/simple correlation) and the size sample (32/64 bits) of the architecture.

Concerning the maximum frequency criteria, we could notice the classification is approximately the same as for the size criteria. The smaller the architecture is, the faster is. Indeed TH-OOK-v2, TH-BPAM-v1, TH-BPAM-v2, and TH-PPM, which are the smallest architectures, are also the faster receivers (frequency of the clock), while, TH-PPM version 2, 3 and 4, are the bigger and the slower architectures. Thus we could say that use 64 bits sample and set up distance estimation block imply an increase of the receiver size and a decrease of the maximum frequency acceptable.

We point out your attention on the fact that, in Time Hopping IR-UWB architecture, the maximum data rate depends on the maximum frequency. We have demonstrated (1) that data rate is function of the frame duration $\left(T_{f}\right)$ and the time slot duration $\left(T_{c}\right)$. Consequently, since $T_{f}$ and $T_{c}$ are expressed in clock period, data rate depends on the maximum frequency. The higher the max frequency is, the higher the data rate is.

The last column of the table 2, summarizes the BER/SNR performance, by proposing a classification according the BER criteria. We obtained thanks to this low level design and simulation the same BER results that with high level Matlab simulation, i.e. TH-PPM proposed a better BER than TH-BPAM, which is better than TH-OOK. Now study the impact of the size sample, the number of channel, the distance estimation, the reconfigurable capability, and the block positioning.

TH-BPAM-v1 and TH-BPAM-v2 allow us to analyze the impact of the block position. Indeed, in the TH-BPAM version, only the TH-discrimination block position change. By comparing the capacities of these two architectures, we could note that they are identical, thus blocks position don't impact the receiver properties.

Concerning the size sample, thanks to TH-OOK-v1 and TH-OOK-v2, you could demonstrate that a change in size sample imply a size increase and a clock speed (consequently data rate) decrease.

TH-PPM version 3 and 4, show that the increase of the channel number on the receiver leads to a decrease of the maximum frequency and an increase of the size. We could obtain the same conclusion, thanks to TH-PPM-v1, TH-PPM-v2 and THPPM-v3, relevant to the impact of the reconfigurability and distance estimation implementation. Nevertheless, distance estimation impact in a higher way the maximum frequency than the implementation of the reconfigurability or the rise of the number of channel. 
In conclusion, the addition of advanced functionality, such as distance estimation, double correlation, multi channel capability leads to size increase and consequently cost and power consumption increase.

This analysis comparative have permitted to deduce some interesting choice in design to optimize the receiver in the WSN context.

\section{Conclusion}

High level and low level modeling, co-design and co-simulation of IR-UWB receiver are presented in this paper. Using Matlab and VHDL software, we could validated, compared, classified distinct receiver architecture in the WSN networks. First, we present TH-PPM, TH-OOK, TH-BPAM IR-UWB concept. Second, we have compared them with respect to the BER versus SNR criteria and WSN constraints at low and high level each time. In particular, our study proves that TH-PPM offers better BER performance than TH-BPAM and TH-OOK systems.

The paper exposes also the impact of the design architecture choice on the respect of the WSN constraints. We introduced the two design way: optimized radio interface versus reconfigurable radio interface. Among our different receiver architecture propositions, we have developed data rate reconfigurable, TH-code reconfigurable and distance estimation capabilities receiver. Each receiver is implemented on FPGA. The co-design Matlab - VHDL software carried out here, allowed us to propose an software-defined radio PHYsical layer. We have developed here a platform for simulation and modeling (before FPGA implantation) at two levels: system level (our IR-UWB Matlab Model) and PHYsical level. We have shared the work between Matlab and VHDL simulator in order to design and emulate the distinct layers (application layer, MAC layer and PHY layer). This platform allows the system codesign, co-simulation and co-performances analysis.

\section{References}

1. I. Opperman, et al., « UWB theory and applications », Wiley 2004.

2. D. Morche, et al., "Vue d'ensemble des architecture RF pour l'UWB », LETI, UWB Summer School, Valence, France, oct. 2006 à l’ESISAR.

3. M.Z. Win, et al., "Impulse radio: how it works", IEEE Communications Letters, Feb 1998.

4. A. Saleh, R. Valenzuela, "A statistical model for indoor multipath propagation", IEEE Journal on selected areas in communications, February 1987.

5. A. Molisch, et al., « IEEE 802.15.4a channel model - final report », IEEE 802.15.4a.

6. LM Aubert, Ph.D. dissertation: "Mise en place d'une couche physique pour les futurs systèmes de radiocommunications hauts débits UWB », INSA Rennes, France, 2005.

7. MG. Di Benedetto, et.al, «(UWB)2: Uncoordinated, Wireless, Baseborn Medium Access for UWB Communication Networks", Mobile Networks and Applications, vol. 10, Oct. 2005.

8. A. Lecointre, "IR-UWB Receiver Architectures Performances on AWGN Channel for Sensor Network Applications", Master dissertation, University of Toulouse, sept. 2006

9. S. Gezici, et al., "Localization via UWB radios”, IEEE Signal Processing, July 2005.

10.J. Mitola III, “Software radio architecture”, Wiley 2000. 